\title{
Cardiac Amyloidosis Is Underdiagnosed in Patients Undergoing Transcatheter Aortic Valve Replacement
}

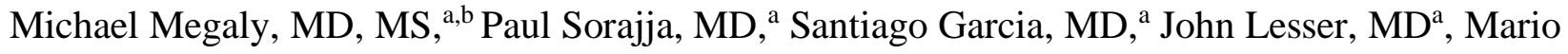
Gössl, MD, PhD, ${ }^{a}$ Matthew Maurer, $\mathrm{MD}^{\mathrm{c}}$, Thomas Treibel, $\mathrm{MD}^{\mathrm{d}}$, João L. Cavalcante, $\mathrm{MD}^{\mathrm{a}}$

a Valve Science Center, Minneapolis Heart Institute, Abbott Northwestern Hospital, Minneapolis, MN, USA.

b Division of Cardiology, Hennepin Healthcare, Minneapolis, MN

c Columbia University Irving Medical Center, New York, NY

d Barts Heart Center and University College of London, London, UK

Short title: Amyloidosis in patients undergoing TAVR

\section{Address for correspondence}

João L. Cavalcante, MD, FACC

Valve Science Center, Minneapolis Heart Institute Foundation, Abbott Northwestern Hospital, Minneapolis, MN, USA.

920 E 28th Street \#300, Minneapolis, Minnesota 55407

E-mail: Joao.Cavalcante@allina.com

Telephone: 612-863-3900 
Word count: 1260 (text)

Keywords: Aortic stenosis, Transcatheter aortic valve replacement, amyloidosis 


\section{Introduction}

Dual pathology of aortic stenosis (AS) and cardiac amyloidosis (CA) is increasingly recognized in our aging population. In hindsight, this may not come as a surprise as AS is the most common valvular disease in the elderly. transthyretin cardiac amyloidosis (ATTR) is common in the elderly with $32 \%$ in autopsy studies of patients $>75$ years with heart failure with preserved EF (HFpEF). ${ }^{1}$

The identification of the dual-pathology can be challenging and requires an index of suspicion, because the two conditions share several common features, including disproportionate left ventricle (LV) mass to electrocardiographic (EKG) voltage ratio and low-flow low-gradient AS. The combination of laboratory testing with non-invasive imaging allows for excellent diagnosis. ${ }^{2}$ Despite the advances in the diagnosis and awareness of this dual pathology, we hypothesized that, in real-world practice, CA remains underdiagnosed in patients undergoing transcatheter aortic valve replacement (TAVR) procedure.

\section{Methods}

We aimed to identify, in the nationwide inpatient sample database (NIS), the percentage of patients undergoing TAVR who also carried the diagnosis of CA. We queried NIS, Healthcare Cost and Utilization Project, from the years 2016 and 2017 using the appropriate International Classification of Diseases (ICD 10) codes to identify patients with severe AS who underwent TAVR. We then identified patients with the diagnosis of CA (codes E852, E854, E853, E8581, E851, E850, E859, E8589, E859 plus code I43). We identified patients who underwent TAVR using the TAVR procedure coded (02RF38H, 02RF38Z). All analyses were conducted using the appropriate weighting, stratifying, and clustering samples following HCUP regulations to 
calculate estimates with a 95\% confidence interval (CI). All statistical analyses were performed using SPSS Statistics for Windows (version 25.0. Armonk, NY: IBM Corp).

\section{Results}

Our cohort included 80,380 weighted discharges for severe AS patients who underwent TAVR without missing data on in-hospital mortality. Within this cohort, 70 patients had the diagnosis of amyloidosis $(0.1 \%, 95 \%$ CI $0 \%$ to $0.2 \%)$, and only 45 patients had the concomitant diagnosis of CA $(<0.1 \%, 95 \%$ CI $0 \%$ to $0.1 \%)$. For all patients undergoing TAVR, the median age was 82 (Interquartile range (IQR): 75-87) years. HFpEF was present in $41.3 \%$ of patients.

In the 45 patients with the current diagnosis of $\mathrm{CA}, 67 \%$ were men, and the median age was 81 (IQR: 79-84) years. Most of the patients were white (77.8\%), and 44.4\% had HFpEF, but only $11 \%$ admitted with acute decompensation of HFpEF. None of the patients had a history of hematologic or solid tumors, and $11 \%$ of them had prior permanent pacemaker implantation (PPMi). Most of these patients were diagnosed in either large $(89 \%)$ or teaching $(100 \%)$ hospitals. The incidence of in-hospital mortality was $11.1 \%$. The incidence of acute kidney injury resulting in hemodialysis was $11.1 \%$, and there was no PPMi or urgent surgery (Figure 1).

\section{Discussion}

Our study shows that in the NIS database of patients receiving TAVR, the prevalence of $\mathrm{CA}$ is two-orders of magnitude lower than in recent screening cohorts. In our analysis, the prevalence was $0.1 \%$ (95\% CI 0-0.1\%) compared to prior clinical reports, which estimated a percentage of $13.9-16 \%$ for the dual pathology. ${ }^{3,4}$ We believe that significant underdiagnosis (US-wide $~ 12,000$ inpatients/year) and poor awareness at that time are likely responsible for our 
observations. Furthermore, these patients were mostly diagnosed in large, teaching hospitals, where one would suspect that a higher level of suspicion and awareness for cardiac amyloidosis would occur. Despite the limitations of our analysis with its observational retrospective nature and the inherent limitations of selection bias and the possible coding errors of the administrative database, including the possibility of under-coding, we believe that our findings demonstrate the lack of awareness of the common dual pathology occurrence. Another limitation of our study is that we performed our analysis in the years 2016 and 2017 when there was limited awareness of the existence of the dual pathology.

A high level of suspicion and awareness is required to identify patients with CA with severe AS undergoing either TAVR or surgical aortic valve replacement, especially in older patients with low-flow low-gradient severe AS. However, this approach using red flags and a high index of suspicision might under estimate the true prevalence of CA in patients with severe AS. A more systematic approach, such as performing bone scintigraphy or cardiac magnetic resonance imaging on all patients with planned aortic valve replacement, might be warranted but this testing strategy needs further investigation. Although the futility of aortic valve replacement in patients with concomitant CA has been debated, recent studies demonstrated that the presence of CA did not significantly affect mortality within two years after TAVR. ${ }^{5}$ Moreover, about 10 $15 \%$ of patients post-TAVR could potentially benefit from new ATTR therapies. Identification of these patients is essential for informed decision making regarding treatment options. It is time to raise awareness for the importance of this dual-pathology as emerging therapeutic options for ATTR to provide an opportunity for sequential treatment. 
Conclusions: Dual pathology of severe AS and CA in patients undergoing TAVR was extensively underdiagnosed in this large inpatient database encompassing the years of 2016 and 2017. A high level of suspicion and awareness is required to identify such patients, and to provide them with an opportunity for sequential treatment.

\section{Acknowledgment}

None

\section{Funding Sources}

None

\section{Declaration of interest}

João L. Cavalcante: Consulting: Abbott Vascular, Boston Scientific, Medtronic, 4Tech, Edwards Lifesciences. Grant support: Edwards Lifesciences, Medtronic, Abbott Vascular, Boston Scientific, Circle Cardiovascular Imaging. Speaker's honoraria: Siemens Healthineers, Medtronic.

Santiago Garcia: Consultant for Surmodics, Osprey Medical, Medtronic, Edwards Lifesciences, and Abbott. Dr. Garcia has received grant support from Edwards Lifesciences and the VA Office of Research and Development.

Paul Sorajja: consulting, speaking for Abbott Vascular, Edwards Lifesciences, Medtronic, and Boston Scientific; equity and consulting for Pipeline Technologies and Admedus 
All other authors have nothing to disclose.

\section{References}

1. Nietlispach F, Webb JG, Ye J, Cheung A, Lichtenstein SV, Carere RG, et al. Pathology of transcatheter valve therapy. JACC Cardiovascular interventions. 2012;5:582-590.

2. Ternacle J, Krapf L, Mohty D, Magne J, Nguyen A, Galat A, et al. Aortic Stenosis and Cardiac Amyloidosis: JACC Review Topic of the Week. J Am Coll Cardiol. 2019;74:2638-2651. 3. Scully PR, Treibel TA, Fontana M, Lloyd G, Mullen M, Pugliese F, et al. Prevalence of cardiac amyloidosis in patients referred for transcatheter aortic valve replacement. J Am Coll Cardiol. 2018;71:463-464.

4. Castano A, Narotsky DL, Hamid N, Khalique OK, Morgenstern R, DeLuca A, et al. Unveiling transthyretin cardiac amyloidosis and its predictors among elderly patients with severe aortic stenosis undergoing transcatheter aortic valve replacement. Eur Heart J. 2017;38:28792887.

5. Scully PR, Patel KP, Treibel TA, Thornton GD, Hughes RK, Chadalavada S, et al. Prevalence and outcome of dual aortic stenosis and cardiac amyloid pathology in patients referred for transcatheter aortic valve implantation. Eur Heart J. 2020. 
Figure legends

Figure 1.

Summary of the study results. 


\section{Cardiac amyloidosis in patients undergoing transcatheter aortic valve relacement}

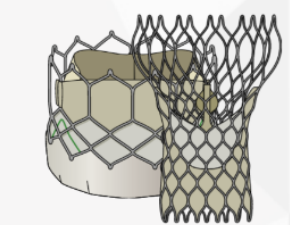

Nationwide inpatient sample database (2016 and 2017)

80,380 discharges with transcatheter aortic valve replacement

Only 45 discharges with cardiac amyloidosis ( $<0.1 \%)$
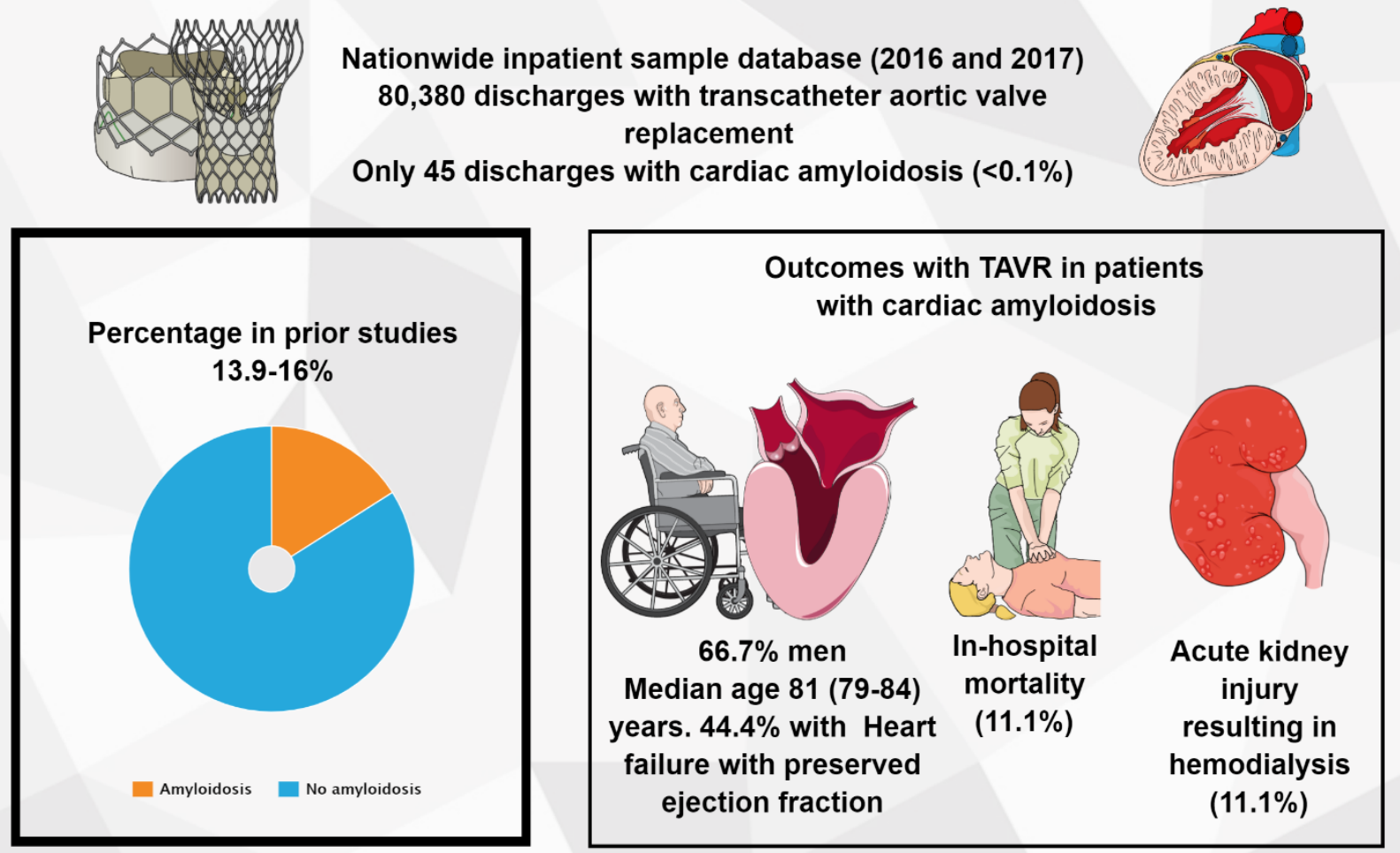

TAVR: transcatheter aortic valve replacement 